\title{
El valor del hacer en la dinámica resiliencia - proceso de reinserción
}

\author{
Marta Lucía Santacruz González*
}

\section{Resumen}

El proyecto que dio lugar a este artículo surge de la experiencia con población reinsertada; dicho proceso se fundamenta en los conceptos acerca de la resiliencia, las características de la población reinsertada y de manera importante en el valor de la actividad, la palabra y la alianza terapéutica dentro de los intentos por lograr en estos individuos la resignificación de su desempeño ocupacional. Se trabaja en su orden, el contexto de esta problemática colombiana, el ser de la persona reinsertada, los aportes del tema de la resiliencia y la dinámica "Reinserción - hacer - resiliencia", para finalizar con una propuesta desde el dominio de estudio de Terapia Ocupacional. Proyecto iniciado en julio del 2003 por la Facultad de Terapia Ocupacional de la Institución Universitaria Escuela Colombiana de Rehabilitación en la fundación "Amor al prójimo y al medio ambiente", que cobija población desmovilizada en proceso de reinsertación a la vida civil.

Palabras clave: reinserción - resiliencia - terapia ocupacional

El presente escrito trata sobre:

La libertad y el encuentro con el sí mismo,

El silencio, la exploración y la creación, como principios de la motivación,

El hacer y la palabra para el encuentro con el entorno,

El vínculo, la pérdida y el apego para el renacimiento, $y$

El desarraigo existencial más allá de lo terrenal.

Por eso escribir sobre: el dolor, la pérdida, el temor y el desapego
Al acompañamiento en el duelo y la celebración de nuevos vínculos,

Vivir el gozo del encuentro con la confianza, para ser capaz de sentir, mirar, tocar, manipular y crear, aceptando la necesidad,

Reorganizar la nada desde el vacío que da la libertad para decidir y elegir desde adentro,

Vivir el desasosiego para resignificar los obje-

* Terapeuta Ocupacional. Especialista en Docencia Universitaria. Docente Facultad de Terapia Ocupacional de la Institución Universitaria Escuela Colombiana de Rehabilitación. 
tos, mediante la ternura y el movimiento que abraza y seduce hasta el cansancio del asombro y el placer,

Entrar en su dolor y su miedo,

Aliarse con sus recuerdos y deseos,

Alcanzar el acercamiento desde la ruptura, y

Acerca de renacer desde el valor de la acción, el propósito y la libertad.

(Santacruz, 2004).

La libertad es un concepto que otorga al ser humano la posibilidad de elegir y direccionar su estilo de vida, valor que en el colombiano actual está trastocado debido a factores de diverso orden, los cuales desafortunadamente giran sobre la problemática generada por el conflicto armado el cual ha dejado numerosas víctimas a diferentes niveles y de manera importante afectando la calidad de vida de la población en general.

Este panorama motiva desde Terapia Ocupacional la búsqueda de alternativas de vida y un mayor bienestar para la persona reinsertada, donde, a partir de la actividad cuidadosamente orientada, la participación del individuo en ocupaciones significativas para él, y el acompañamiento en el proceso de reorganización de su desempeño ocupacional, se establecen caminos posibles dentro del paradigma de la Resiliencia. Este término es definido por Boris Cyrulnik (1999), como la capacidad del ser humano de resistir y aprender a vivir ante una situación extrema, que como proceso de destrucción de la vida, encierra en forma paradójica un potencial de vida; destrucción sentida por las personas reinsertadas que se enfrentan a un entorno desconocido y a su posible capacidad de renacer.

En relación con esto, el Estado colombiano con el Ministerio del Interior, ha suscrito acuerdos de paz con nueve movimientos guerrilleros. Como producto de estas negociaciones 4.715 personas que han dejado las armas de manera voluntaria y colectiva, y se han acogido a los programas de reinserción que ofrece el Estado. Los subversivos que han dejado de manera individual y voluntaria las armas son amparados por el Decreto 1385 de 1994, bajo los conceptos de desarme, desmovilización y reintegración o reinserción, relacionados con la aplicación de programas de compensación monetaria, entrenamiento, capacitación, generación de ingresos y otros beneficios, que buscan garantizar la reinserción social y económica de los excombatientes y sus familias.

Este proceso de reinserción hace parte del cumplimiento de los acuerdos de paz y busca que las personas directamente afectadas logren nuevos proyectos de vida con respaldo del gobierno y entidades que trabajen con esta población, como las Organizaciones No Gubernamentales (ONG), cuyo objetivo, es lograr que estas personas se reincorporen a la vida social y laboral. Estas entidades a través de albergues brindan la oportunidad a esta población de mejorar su situación, enfocar nuevas expectativas de su proyecto de vida e incorporarse a la vida civil.

\section{Una mirada al desempeño ocupacional de la persona desmovilizada}

La persona que asume el rol social de desmovilizado posee comportamientos socioculturales, psicológicos, espirituales y físicos, relacionados directamente con su experiencia de vida en grupos armados, su herencia cultural y su propio proceso de desarrollo biopsicosocial. Igualmente, la vivencia como sujeto en camino de asumir una nueva forma de existencia, le demanda nuevas estrategias y competencias personales para manejar los sentimientos de desarraigo, estrés, desconfianza, pérdida, soledad, culpa, temor, vacío y los relacionados con los conflictos de identidad que este tipo de cambios generan en el individuo.

Al respecto, en el proyecto colectivo de la $\mathrm{Fa}-$ cultad de Psicología de la Pontificia Universidad Javeriana (2002), liderado por Zoraida Martínez, 
Liliana Muñoz y cinco estudiantes, se describe un perfil psicológico de la persona desplazada por la violencia y que se aplica a personas desmovilizadas. El estudio refiere que:

"estas personas se encuentran en situación de desventaja psicológica por cuanto ha habido una lesión a su equilibrio psíquico, con sentimientos de culpa y desestructuración de los vínculos afectivos. Existe confusión de la identidad, pérdida del significado de la vida en común, caos dentro de la familia, violencia social, dolor y angustia, con trastornos desadaptativos, síndromes depresivos, ansiosos y de estrés postraumático". (Pág. 25).

Lo anterior, afirman las autoras de dicho estudio, tiene unas características que lesionan la condición humana porque:

"genera una situación de desarraigo, falta de claridad sobre su ser social con confusión de la imagen de sí mismo con la imagen que la sociedad proyecta sobre él. En la mayoría de los casos se pierden los grupos de referencia y se desarticulan los de pertenencia, esto constituye la base de destrucción de la estructura de identidad personal y familiar, se establece la soledad física y afectiva, vivida como muerte en vida. La desaparición de la comunidad referida, incluso solamente el área geográfica, genera sentimientos de confusión, humillación, desesperanza, culpa; por su parte, el aislamiento, el ocultamiento físico, la vergüenza, la desmotivación y la incapacidad de reconstruir su proyecto vital, hacen parte del mecanismo psicológico que se presenta cuando un ser humano ha experimentado condiciones infrahumanas". (Pág. 26).

Estos sentimientos de desarraigo, la reubicación, la búsqueda de nuevas oportunidades en las grandes ciudades, afectan a la sociedad y a la persona víctima del conflicto armado.

Como consecuencia de dicha situación, se establece un imbalance en sus vidas y una disfunción en su desempeño ocupacional, con una problemática psicosocial, generada por causas socioculturales que afectan de manera importante la motivación interna, la identidad, la confianza, la dimensión relacional, el sentido de pertenencia, la capacidad de utilizar las habilidades en un desempeño ocupacional competente, el sentido de vida y la proyección de un proceso vital propio. En este orden de ideas, la situación de la persona desmovilizada es compleja e involucra, además, de los desajustes psicológicos, los de tipo laboral, social y de valores, ya que en general, son personas de origen rural quienes se ven obligadas a interiorizar nuevos estilos de vida y adaptarse a una nueva cultura. Esta situación afecta las diferentes dimensiones del ser humano, las cuales deben ser atendidas con una visión holística, transcultural, relacional, del valor del hacer, la libertad de elección y los fundamentos de la resiliencia, con la meta de generar motivación, facilitar sus procesos de cambio, promover prospecciones laborales, personales y un proyecto de vida familiar.

En este panorama ocupacional, se hace necesario analizar los factores a que está sometida esta población, pues, como consecuencia del desplazamiento forzado, la persona sufre cambios en su estilo de vida en términos de cotidianidad, ya que se ve obligada a cambiar de ambiente a un entorno con demandas culturales, sociales, económicas y laborales diferentes a las de su anterior vida, esto conlleva cambios abruptos en su desempeño ocupacional, en los procesos de autocuidado, trabajo, esparcimiento y en las expectativas de vida, por tanto, el proceso de readaptación a un nuevo estilo de vida posee un curso lento, sujeto a la aceptación consciente de las actuales circunstancias de vida y de las oportunidades que le ofrece su nuevo entorno.

En este contexto y como parte del presente estudio se hace necesario caracterizar la población en proceso de reinserción teniendo como referente conceptual el Modelo del desempeño Ocupa- 
cional Realizante de Terapia Ocupacional, (Trujillo 1995), el cual se define como:

\begin{abstract}
"una forma humanista existencial de concebir el actuar ocupacional, a través del cual la persona tiende a su realización como el propósito más alto de las ocupaciones de autocuidado, juego y trabajo y facilitado por la organización, integración y productividad". (Pág. 11).
\end{abstract}

En este sentido, esta población se caracteriza así: en cuanto a los procesos humanos, predomina población de origen rural, en edades que corresponde al adulto joven y medio, edad de ingreso a la guerrilla entre los 10 y 17 años, mayoría hombres, con nivel de escolaridad por debajo de la básica primaria, solteros. Ingresan a la guerrilla de manera forzosa y por razones económicas. Las causas de su desmovilización son el maltrato, temor a la muerte y la falta de remuneración económica. Se evidencia dificultades sensorio-motoras en relación con la capacidad de planeamiento y destrezas, en ocasiones con presencia de temblor, causado por la exposición a estímulos vibracionales como el manejo del fusil y deprivación ambiental. En el desempeño cognoscitivo, además, del alto índice de analfabetismo, presentan bajos niveles para la solución de problemas, simbolización y creación. Por otra parte, el comportamiento socio-emocional se muestra como el más afectado, ya que presentan procesos pobres de vinculación, desconfianza, temor, desapego y limitaciones para establecer relaciones profundas con otros, pues cada acercamiento se concibe como posible amenaza para su Yo. Los sentimientos de desarraigo y culpa afectan su motivación, identidad y sentido de pertenencia, e inciden de manera importante en su autoestima, en la capacidad de pedir ayuda y expresar emociones. Estar en un entorno desconocido y la sensación de no poseer el control les genera imbalance en el ser, el actuar y el sentir, con la consecuente dificultad para explorar, sentir, crear y buscar la autorrealización.
En los procesos ocupacionales, el cambio en su forma de vida conlleva la alteración en el estilo del desempeño ocupacional, asumiendo actitudes de rebeldía y oposición ante las normas, límites y las nuevas rutinas que su actual entorno les demanda, lo que puede hacer su proceso de incorporación y convivencia más difícil. Algunos asumen comportamientos pasivos ante las actividades cotidianas, afirmando que el gobierno es responsable de su futuro, por lo tanto le demandan la solución a todos sus problemas. En cuanto a los hábitos y rutinas se destaca la organización inadecuada de estas conductas, esto se apoya en el hecho de que no tienen horarios por cumplir, ni actividades fijas por realizar, con desorganización en las actividades de autocuidado, en la estructuración del tiempo y de manera importante en el interés por realizar tareas productivas, ya que la mayoría, sólo espera la remuneración económica del Estado.

En referencia al contexto físico, temporal y sociocultural, los sentimientos de desarraigo afectan su identidad y sentido de pertenencia con cambios en sus motivaciones ocupacionales, suscribiendo éstas al reconocimiento del medio circundante, identificación de las demandas sociales y económicas del hábitat urbano y exploración de alternativas viables para la supervivencia inmediata, siendo la seguridad física una de sus mayores preocupaciones; sin embargo, la desvinculación cultural produce alteración en la motivación para realizar nuevas actividades y participar en programas de capacitación, ya que no llenan sus expectativas e intereses, y sólo representan un requisito para la libertad. Además, poseen habilidades poco efectivas para el desempeño ocupacional propio de la ciudad, puesto que cuentan con las que demanda un medio rural; por dicha razón, al asentarse en el medio urbano se empobrece la percepción de metas futuras.

Esta descripción desde el Modelo del Desempeño Ocupacional Realizante de la persona en 
proceso de reinsertación a la vida civil muestra la alteración de sus habilidades básicas para el desempeño competente en las actividades ocupacionales cotidianas con la consecuente carencia de propósito en su actuar ocupacional y por ende sentimientos de vacío en lo que atañe a su sentido de realización; no obstante, cabe resaltar que cuentan con elementos sanos dentro de su personalidad, como la capacidad de asombro, de vivir la lúdica y recibir del otro cuando perciben que éste no le va a hacer daño, actuando desde su ingenuidad, necesidad de logro, de sus núcleos infantiles y su instinto de supervivencia, así como de las cualidades personales que permiten pensar que estas personas, a pesar de tener en su interior sentimientos desfavorables para un mejor estar, poseen dentro de sí la posibilidad de ser partícipes de su acto resiliente y lograr asumir un nuevo estilo de vida ocupacional.

\section{Vacío existencial y el acto resiliente en población desmovilizada}

En este contexto, para Balegno y cols. (2002), la resilencia se define como:

"la capacidad que tiene todo ser humano a pesar de sus dificultades personales (físicas, mentales y psíquicas) para intuir y crear en circunstancias desfavorables o en un entorno destructor, respuestas que le hacen posible no dejarse atrapar por las circunstancias y dificultades personales ni por las condiciones del entorno, sino por el contrario, proponer y realizar comportamientos que le permitan una vida con menos sufrimiento y encontrar un lugar en su medio ambiente, lo cual, le permite desarrollar sus capacidades y anhelos". (Pág. 209).

Para este autor, sufrir y ser víctima no son sinónimos, refiriendo que toda víctima es un ser humano que sufre, pero un ser humano que sufre puede o no considerarse a sí mismo o ser considerado como víctima. Entonces, ¿la persona desmovilizada es una víctima que sufre? Este cuestionamiento puede tener dos miradas: por un lado, sufre por las circunstancias que debió elegir para ofrecerse un mejor estar en la vida, o bien, puede ser un ser humano quien se victimiza como defensa de los sentimientos de culpa que le generan sus actos pasados.

Por tanto, para Balegno (2002), el panorama de la resiliencia demanda una mirada diferente sobre el sujeto que está sufriendo debido a actos violentos. Esto implica al terapeuta tener una nueva capacidad de escucha e investigar nuevas formas de intervención, es decir, consiste en un proceso de desvictimizar a la víctima. Sin embargo, esta capacidad de renacer y encontrar el camino para el cambio, no se da de igual forma en todas las personas, dado que intervienen componentes psicológicos, circunstanciales y características del entorno.

En este sentido, Colmenares en el mismo texto de Belegno (2002), refiere que:

"existen personas con una asombrosa capacidad para trascender exitosamente graves daños y conservar el placer y la alegría de vivir, con el poder para que, en medio de la violencia y de las peores calamidades, puedan definir a partir de sí mismos, el lugar y sentido de su experiencia trascendiendo la circunstancia y conservando valores éticos de supervivencia y desarrollo que les permitan mantener y construir sus sueños, esto lleva a que la persona supere las circunstancias en que los hechos se produjeron, para estudiar el lugar que toma el juego de los factores externos en las posibilidades o imposibilidades de un acto resiliente, donde son igual de importantes las responsabilidades individuales y sociales". (Pág. 84).

Al respecto, Cyrulnik en el mismo texto de Balegno (2002), afirma que:

"el comportamiento resiliente como tal es una respuesta para salvaguardar el sentido de la propia vida como principio de supervivencia en situaciones o hechos que comprometen gravemente esta posibilidad de supervivencia y que ocasiona daños severos a la vida psíquica", (pág. 183), 
Este principio se encuentra en la población desmovilizada, a pesar de su dificultad para encontrar una representación del sí mismo en sus relaciones, su hacer, su necesidad de autorrealización y en el nuevo entorno que lo contiene.

En esta medida, trabajar el acto resiliente en el sujeto en proceso de reinserción, es urgente y posible, en la medida que su instinto de supervivencia se presenta de manera contundente y la necesidad de reorganizar su identidad se da dentro de su cotidianidad con la búsqueda de apegos que le ofrezcan seguridad y nuevas posibilidades de relaciones significativas, sin embargo, esta reestructuración de un estilo de vida, requiere de un proceso terapéutico donde la escucha, el acompañamiento, el apoyo y la actividad, actúen sobre la capacidad de la persona para tomar conciencia de su situación y asumir la responsabilidad de su proceso de cambio y su nueva vida.

\section{El hacer dentro del acto resiliente}

La filosofía y metodología de este proyecto se fundamenta en el cuerpo teórico-práctico de la Terapia Ocupacional, la Teoría de la Vinculación de Bowlby (1951) y Ainsworth (1977), la Teoría de los Objetos Internos de Freud (1938), el Modelo del Desempeño Ocupacional Realizante de Trujillo (1995), el valor terapéutico del hacer y los principios de la resiliencia en la intervención terapéutica, articulados bajo las siguientes premisas y pasos de aplicación:

Primera fase: creación de la alianza terapéutica, vínculos seguros y confianza en el otro. Esta fase es esencial porque la ruptura temprana o violenta del apego (antes o durante la adolescencia) convierte los objetos interiorizados como buenos en objetos externos amenazantes, entonces, no se vivencian lazos seguros y el entorno desconocido no ofrece seguridad, lo que afecta la exploración, independencia, seguridad y confianza para establecer nuevos vínculos. La conducta de vinculación caracteriza a los seres humanos de la cuna a la tumba, donde, los vínculos o lazos emocionales relativamente permanentes hacia individuos particulares, figuras paternas o representantes simbólicos de las figuras primarias que proporcionen una base segura en situaciones que son desconocidas y atemorizantes, son de particular importancia, porque actúan como un prototipo para todas las relaciones presentes y posteriores, de manera importante, ofrecen la base para interiorizar objetos internos buenos que remplacen los objetos muertos que las experiencias de soledad y vivencias violentas han depositado en el individuo desmovilizado. De ahí, que el conocimiento de la importancia de la vinculación y de su pérdida o trastorno es necesaria para comprender la manera en que la experiencia temprana puede afectar el manejo de experiencias posteriores y para contextualizar la alianza terapéutica.

Segunda fase: en este momento el hacer ocurre dentro de un contexto lúdico como forma de acercamiento y exploración del desempeño ocupacional. Aquí se parte del hecho que el juego es la primera actividad con propósito y significado que realiza el ser humano, ya que es un tipo de acción que da placer, gratificación, relajación física y psíquica, además, ofrece un ambiente seguro que posibilita reparar el Yo, da la posibilidad de ser flexibles para adquirir sentimientos de control, desarrollar habilidades y sentido de logro, así como, encontrarse a sí mismo a partir del encuentro con los objetos y los demás. Desde esta perspectiva, se asume la actividad lúdica dentro de este proyecto como una actividad motivante ya que el sujeto siente que la controla, que no es amenazante y ofrece confianza para actuar sobre el entorno, que posibilita la desinhibición y por tanto, la persona se muestra en su realidad. De manera importante, es un acto que involucra al individuo en forma integral, pues ofrece información completa sobre la calidad de su desempeño ocupacional y el espacio para que el sujeto realice su narrativa personal. 
Tercera fase: aquí prevalecen la palabra y la alianza terapéutica como generadoras de vínculos, motivación, autoconocimiento y el camino hacia el acto resiliente. Los primeros vínculos en el ciclo vital parten del estímulo dado por la palabra, la voz y su referente auditivo. Balegno (2002) considera que el papel del terapeuta es acercarse al sufrimiento de la persona mediante un tipo de escucha diferente, ser observador y no tener prejuicios, para comprender cómo es la mirada del sujeto hacia el entorno y hacia sí mismo, de este modo, la escucha resiliente consiste en identificar las manifestaciones positivas del comportamiento y hacer un inventario de dichas cualidades, estar alerta a la percepción del usuario sobre su sufrimiento e interpretar sus intuiciones resilientes, acompañarlo en la construcción de su acto resiliente, apoyar sus cualidades, promover la toma de conciencia del sí mismo y su situación, así como, de su responsabilidad dentro del proceso de cambio.

Cuarta fase: denominada del simbolismo en el hacer para la creación de objetos internos buenos, vínculos con el sí mismo, los otros y el entorno, donde se parte que para Freud (1938), el Yo es ante todo corporal y luego psíquico, de ahí, que para la incorporación de los objetos, sus representantes internos el desarrollo de vínculos sean necesarios los elementos del sistema nervioso, sensitivos, de asociación, de movimientos y los conservadores de la memoria, sin embargo, sin la presencia del Yo no podrían producirse los objetos internos. Es decir, las primeras incorporaciones son de carácter vital, como satisfacer las necesidades fisiológicas primarias, el contacto táctil y visual con la presencia del objeto que ofrece seguridad, luego, aparece el movimiento y el objeto que se toca y desea, la manipulación que da vitalidad posibilita apropiarse del objeto y da sentido de control, el objeto como espacio entre la fantasía y la realidad, y por último la creación que permite la diferencia del mundo vital y no vital, precursor del acto resiliente.
Quinta fase: la resiliencia desde los actos lúdico y creativo, como elementos de supervivencia, recuperación de la identidad y apropiación del proyecto vital. En esta fase se enfatiza en la libertad de elección, el ser humano asume la exploración, la manipulación y la construcción de procesos que le generen gratificación, vive el asombro ante el encuentro con sus posibilidades en el entorno, adquiere motivación y satisfacción en las relaciones con otros, gana confianza en la palabra que surge como necesidad de liberación y acercamiento, en el movimiento que desinhibe al ser y la creación de nuevos vínculos que le ofrecen seguridad y sentido de control.

\section{Propuesta desde Terapia Ocupacional para la intervención terapéutica con población desmovilizada en proceso de reinsertación a la vida civil}

Más que una propuesta de orden técnico, el presente proyecto contiene una visión filosófica y cualitativa para el abordaje de población desmovilizada, mediante los valores del hacer y la relación terapéutica en la alianza para la actividad, el sujeto y el entorno. Esto se fundamenta en los conceptos del vínculo y su importancia en el acto resiliente según Peloquín (2002) para quien el carácter integrador de la Terapia Ocupacional parte de la visión y la capacidad del terapeuta para llegar al corazón del usuario, a partir de la acción de sus manos, ya que, es realmente en el corazón donde sucede la curación, de ahí, la competencia para curar, cuidar y dar oportunidades para mayor sentido de competencia, integrando el propósito terapéutico y el sentido personal. Esta autora afirma el hecho de que este profesional interviene en la curación de mentes, cuerpos y almas enfermas por alguna circunstancia vital.

En este contexto, para Peloquín (2002):

"la profesión elabora su práctica sobre el significado de la ocupación en el trabajo de vivir, donde los componentes emocionales, espiritua- 
les y morales del Yo, propician la participación del afecto, los valores, la pasión y lo más profundo de la persona renovando por medio del hacer, lo esencial de su vida como esencia que habita en el corazón, y las manos como el instrumento por el que se toca, controla, se siente, manipula y se ejecutan tareas productivas para el Yo. El valor del hacer, la actuación experimentada y la fabricación con significado, permite al sujeto trascender su visión de actividad y ocupación. Esto, compromete al Terapeuta Ocupacional a ir más allá de su visión de desarrollar habilidades, ir hacia la conexión con el usuario mediante el acto de escuchar, cuidar, facilitar y acompañar en el encuentro con ocupaciones significantes, donde su mano es el medio que comunica con el resultado real". (Pág. 518).

La anterior aproximación filosófica, retomada de manera importante dentro de este proyecto con personas desmovilizadas, se complementa con una aproximación humanista y psicodinámica en términos de libertad, maduración, alianza terapéutica y graduación de la actividad, es decir, el acercamiento del usuario se propone desde su grado de confianza, su nivel de maduración interna, sus intereses y los beneficios de la actividad lúdica en el desarrollo socio-emocional del ser humano, todo dentro de un contexto relacional de contención, acompañamiento y escenarios de experiencia ocupacional dados al sujeto de manera gradual y motivante, con el fin de que afiance sentimientos de logro, sentido y competencia.

En otros términos, el objetivo de competencia ocupacional en estos usuarios, implica remontarse a la visión de ser humano, su naturaleza ocupacional y su desempeño ocupacional, concepto que hace referencia al sujeto que piensa, siente y actúa a partir de su motivación interna para realizar ocupaciones que le ofrezcan sentido. En esta medida, para Terapia Ocupacional, el individuo como ser único y la razón de su hacer, se constituye en un ser activo cuyo desarrollo está influenciado por el uso de su cuerpo-mente y espíritu en actividades con propósito, que a su vez influyen su identidad, sentido de pertenencia, su realización personal y beneficia su salud integral y su entorno.

Desde esta perspectiva, la profesión se interesa por comprender e intervenir en la solución de problemas que afectan el desempeño ocupacional del ser humano, partiendo de principios filosóficos referentes a "el hacer da propósito a la existencia del individuo", aplicable en los casos en que su motivación intrínseca ha sido afectada, limitando su capacidad de participación, convivencia y autorrealización.

En relación con lo anterior, Terapia Ocupacional juega un papel importante en su intervención con población desmovilizada, ya que cuenta con los medios necesarios para determinar los aspectos vulnerables en esta población y enfoca sus recursos para intervenir efectivamente por medio de estrategias ocupacionales orientadas al beneficio del desempeño psicosocial de la población y su capacidad para desempeñar roles ocupacionales, como las actividades lúdicas y proyectivas, donde la alianza terapéutica cumple una función básica.

Según Cuéllar (2000), la dimensión ocupacional de exguerrilleros está afectada por el impacto de esta experiencia en su área social y emocional, con desajustes e interrupciones en los procesos ocupacionales. $\mathrm{Al}$ respecto anota:

"el Terapeuta Ocupacional, trabaja la recons-
trucción de la historia ocupacional y el auto-
rreconocimiento que de ella haga el exguerri-
llero, como medios para la valorización de la
ocupación en sus diferentes etapas y facetas,
como proceso productivo continuo y conexo,
a través del cual se desarrollan habilidades, se
acumulan experiencias y se adquieren cono-
cimientos para alcanzar mayores niveles de
complejidad en el futuro desempeño ocupa-
cional". (Pág. 31).

En otros términos, la persona desmovilizada debe reconstruir su proyecto de vida y apropiarse de un nuevo entorno, que le produce descon- 
fianza y temor, situación que le exige fortalezas personales para afrontar de manera organizada, integrada, productiva y realizante su transición a un nuevo estilo de vida y de desempeño ocupacional.

Los logros obtenidos con el presente proceso son: establecer una alianza terapéutica de confian$\mathrm{za}$, seguridad y acompañamiento que se ha reflejado en acercamiento, motivación, exploración, identificación de intereses, habilidades y limitaciones, aumento de tolerancia a las actividades, aproximación a la lectoescritura, mayor capacidad para crear, expresar y comunicar elementos internos, y de manera importante, el hacer y el sentirse productivos mediante la realización de una actividad. Este es el baluarte para que logren sanar aspectos de su personalidad y enfrentarse a la construcción de su proyecto vital.

Por tanto, se considera que la aproximación a este tipo de población debe realizarse mediante una relación de escucha, libertad, acompañamiento y contención con la actividad lúdica y proyectiva como vivencia de exploración del Sí mismo, no amenazante, relajante, que propicia sentido de logro y congrega grupos. Estas estrategias son válidas para lograr el acercamiento del usuario, su confianza y motivación para la actividad, para propiciar espacios de comunicación y generar vínculos fundamentados en la seguridad y la aceptación, y de manera importante, para reafirmar el valor del hacer como espacio de curación y resiliencia en este tipo de población.

En conclusión, la experiencia que ha sido cuidadosamente seleccionada, graduada y adaptada teniendo en cuenta el contexto donde ocurre, debe dar origen a una nueva fase del proceso, encaminada a trabajar el proyecto de vida en esta población, dentro de la cual se han encontrado núcleos sanos como la capacidad de renacer, asombrarse, gratificarse, crear, confiar, sobrevivir y resignificar su existencia, los cuales se constituyen el punto de partida para que cada individuo dé paso a su capacidad resiliente; sin embargo, surge la siguiente pregunta ¿está la sociedad lista para acompañarlos y contenerlos en su proceso de reinserción y resiliencia?

\section{Referencias}

Balegno, L.; Cyrulnik, B.; Manciaux, M.; Sánchez, E.; Colmenares, M.E. \& Olaya, M. 2002. La resiliencia desvictimizar a la víctima. Casa Editorial Rafue, Colombia.

Cuéllar Patricia. 2000. Una propuesta de atención sociolaboral para exgerrilleros, Revista Ocupación Humana. Asociación Colombiana de Terapia Ocupacional. Bogotá. 8 (3): $24-31$.

Facultad de Psicología de la Pontificia Universidad Javeriana. 2002. Desplazamiento interno y atención psicosocial: el reto de reinventar la vida. Pontificia Universidad Javeriana. Bogotá, 16-8.

Gómez, Marisol; Torres, Sara; Rodríguez, María Teresa. 1999. Caracterización del comportamiento ocupacional de personas desplazadas por la violencia. Revista Ocupación Humana. Asociación Colombiana de Terapia Ocupacional, 8(2): 13-31.

Gross, R. 1991. Desarrollo psicológico. Editorial MacGraw-Hill.

Peloquin, S. 2002. Reclaiming the vision of reaching for heart as well as hands. The American Journal of Occupational Therapy. 56(5): 517-25.

RosAs, P. 2003. Soluciones económicas para los desplazados en Colombia. Konrad, Adenauer. Bogotá.

Santacruz, Martha. 2003. Salud mental, competencia ocupacional y calidad de vida, una experiencia. Institución Universitaria Escuela Colombiana de Rehabilitación. Bogotá. 
Turriago, G.; Bustamante, J. 2003. Estudio de los procesos de reinserción en Colombia 19911998. Alfaomega. Ideas para la paz.

Trujillo, A. 1995. Desempeño ocupacional realizante: un modelo sobre los propósitos de la terapia ocupacional. Revista Ocupación Humana. Asociación Colombiana de Terapia Ocupacional. Bogotá, 6 (2).

ZuBIRÍA, R. 1996. Muerte y psicoanálisis. Editorial Grijalbo, Bogotá. 